\title{
Evaluación biomecánica de reparación de roturas del tendón patelar: ¿Es mejor doble Bunnell paralelo o perpendicular?
}

\section{Biomechanical Comparison of Two Patellar Tendon Repair Techniques: Is Double Bunnell Better Parallel or Perpendicular?}

\author{
Alex Vaisman ${ }^{1}$ Rodrigo Guiloff ${ }^{2}$ Pablo Uauy ${ }^{3}$ Rodolfo Taborga ${ }^{3}$ Nikola Abello ${ }^{3}$ Jorge Joannon ${ }^{3}$ \\ Rodrigo Guzmán ${ }^{4}$ Rafael Calvo ${ }^{1}$ David Figueroa ${ }^{1}$ \\ ${ }^{1}$ Departamento de Ortopedia y Traumatología, Facultad de Medicina, \\ Clínica Alemana Universidad del Desarrollo, Santiago, Chile \\ Address for correspondence Dr. Alex Vaisman, MD, Departamento de \\ Ortopedia y Traumatología, Facultad de Medicina Clínica Alemana \\ 2 Médico Residente de Ortopedia y Traumatología, Facultad de \\ Universidad del Desarrollo, Avda. Vitacura 5951, Vitacura, Santiago, \\ Medicina Clínica Alemana Universidad del Desarrollo, \\ Chile (e-mail: avaismanb@hotmail.com). \\ Santiago, Chile \\ ${ }^{3}$ Alumno, Facultad de Medicina Clínica Alemana Universidad del \\ Desarrollo, Santiago, Chile \\ ${ }^{4}$ Kinesiólogo, Laboratorio de Biomecánica Universidad de Los Andes, \\ Santiago, Chile \\ Rev Chil Ortop Traumatol 2017;58:78-83.
}

\begin{abstract}
Resumen
Introducción En tendón patelar, se ha probado que agregar un segundo punto Bunnell dispuesto perpendicular al punto clásico, es más resistente, sin embargo, esa técnica dispuesta paralelamente no ha sido evaluada.

Propósito Comparar la resistencia entre la técnica doble Bunnell dispuesta en perpendicular y en paralela.

Método 28 muestras frescas de tendones patelares porcinos, asignadas aleatoriamente a 2 grupos. Grupo 1: 14 muestras reparadas con doble Bunnell perpendicular. Grupo 2: 14 muestras reparadas con doble Bunnell paralelo. Todas realizadas con la misma sutura ultrarresistente. Con una máquina computarizada de tracción, se aplicó tracción axial continua hasta el fallo. Se registró el punto máximo de fallo (PMF) y el modelo de falla mecánica. Análisis estadístico con t-student $(p<0,05)$. Resultados Grupo 1: PMF $=342 \pm 50,7$ Newton. Todas las muestras sufrieron

Palabras Clave

- reparación tendínea

- bunnell

- resistencia biomecánica avulsión central, preservando fibras perimetrales. Grupo 2: PMF $=358 \pm 34,1$ Newton. Todas las muestras sufrieron un split longitudinal del tendón. Sin diferencia significativa entre los grupos $(p=0,340)$.

Conclusión Este estudio no demostró diferencias significativas en la resistencia de una técnica de doble Bunnell paralelo versus perpendicular, aplicada en un modelo experimental de tendón patelar.
\end{abstract}

received

September 23, 2017

accepted

November 23, 2017
DOI https://doi.org/

10.1055/s-0037-1615256. ISSN $0716-4548$.
Copyright @ 2017 by Thieme Revinter

Publicações Ltda, Rio de Janeiro, Brazil
License terms

(c) $(1) \$$ 
Relevancia Clínica Ambas técnicas tienen propiedades biomecánicas similares y dejan un remanente de tejido tendinoso luego del fallo que permitiría una segunda reparación directa, potencialmente sin necesidad de requerir el uso de injertos.

Nivel de Evidencia Estudio de Laboratorio Controlado - Nivel IV.

\section{Abstract}

\section{Keywords}

- tendon repair

- bunnell

- biomechanical strength
Introduction In the patellar tendon, a second Bunnell stitch arranged perpendicular to the classic configuration has increased the resistance of the repair, however, the effect of a double parallel technique has not been evaluated.

Purpose To compare the resistance between a patellar tendon repair with Double Bunnell technique arranged in parallel or perpendicular.

Method 28 fresh porcine patellar tendon samples were randomly assigned to 2 groups. Group 1: 14 samples repaired with a perpendicular Double Bunnell technique. Group 2: 14 samples repaired with a parallel Double Bunnell technique. All performed with the same ultraresistant suture. The samples were fixed to a computerized traction machine, applying continuous axial traction, until failure. The ultimate failure point (UFP) and the mechanical failure model were recorded. Statistical analysis included students t-test $(p<0.05)$.

Results Group 1: UFP $=342 \pm 50.7$ Newton. All samples suffered a central avulsion, preserving the perimetral tendon fibers. Group 2: UFP $=358 \pm 34.1$ Newton. All samples suffered a longitudinal split of the tendon. No significant difference was found among groups $(p=0.340)$.

Conclusion This biomechanical study showed no significant differences in the strength of a tendon repair using a parallel versus a perpendicular double Bunnell technique.

Clinical Relevance Both techniques have similar biomechanical properties and leave a remnant of tendon tissue after the failure that would allow a second direct repair, potentially without requiring the use of grafts.

Level of Evidence Laboratory Controlled Study - Level IV.

\section{Introducción}

La reparación quirúrgica por medio de suturas, es el tratamiento de elección ante una rotura total o parcial del tendón patelar asociada a un déficit de la funcionalidad extensora de la rodilla. ${ }^{1,2}$ Series de casos han demostrado entre $70-100 \%$ de excelentes y buenos resultados, alcanzando rangos de movilidad funcionales y fuerza de cuádriceps simétrica con respecto al lado no lesionado. ${ }^{3-6}$

Existen distintas técnicas de reparación incluyendo: suturas término-terminal entre los cabos, suturas a través de túneles trans-óseos y suturas con anclas patelares. No existe consenso sobre la mejor forma de reparación, pues la evidencia comparativa actual entre los distintos métodos es escasa. ${ }^{1,6,7}$ Por lo demás, independiente del método escogido, existen diferentes configuraciones de suturas para lograr la reparación. Dentro de ellas, destaca la técnica de Bunnell, popular en nuestro medio, siendo validada en lesiones de tendón patelar. ${ }^{7}$

Estudios de reparación tendínea han demostrado que agregar una segunda sutura incrementa la resistencia de la reparación, lo que favorecería una rehabilitación acelerada, tanto en extremidad superior con tendones flexores ${ }^{8-12}$ como en extremidad inferior en tendón de Aquiles. ${ }^{13-16}$ Respecto al tendón patelar, en un estudio biomecánico previo, demostramos con éxito esta premisa: agregar un segundo punto Bunnell dispuesto perpendicular al punto clásico aumenta la resistencia de la reparación, ${ }^{17}$ sin embargo, el efecto de esa técnica doble dispuesta paralelamente, no ha sido evaluada, generando la duda si esta mayor resistencia está dada por el número de suturas, o por la disposición espacial de esas.

El propósito de este estudio, es comparar la resistencia entre técnica doble Bunnell dispuesta en perpendicular y en paralela en un modelo de tendón patelar.

La hipótesis de trabajo es que una disposición perpendicular es más resistente al fallo que una disposición paralela debido a que aumenta el área de contacto y proporciona sostén ante la eventual falla de una de las 2 suturas, generando un anclaje de una sutura sobre la otra.

\section{Materiales y Métodos}

\section{Muestras}

Se incluyeron 28 muestras frescas de rodilla porcina $(3 \mathrm{~cm}$ proximal a la patela y $2 \mathrm{~cm}$ distal a la tuberosidad anterior de la tibia). Todos los animales tenían entre 4 a 6 meses de edad, 
Tabla 1 Datos Morfométricos de las muestras del estudio

\begin{tabular}{|l|l|l|l|}
\hline & $\begin{array}{l}\text { Doble Bunnell } \\
\text { Perpendicular } \\
(\mathrm{mm})^{*}\end{array}$ & $\begin{array}{l}\text { Doble Bunnell } \\
\text { Paralelo } \\
(\mathrm{mm})^{*}\end{array}$ & $\begin{array}{l}\text { Comparación } \\
\text { Estadistica }\end{array}$ \\
\hline $\mathrm{n}$ & 14 & 14 & \\
\hline Largo & $45 \pm 0$ & $45 \pm 0$ & \\
\hline Grosor & $7,79 \pm 1,37$ & $7,50 \pm 1,45$ & $\begin{array}{l}\mathrm{t}=0,535 \\
p=0,597\end{array}$ \\
\hline Ancho & $16,4 \pm 1,74$ & $16,4 \pm 1,60$ & $\begin{array}{l}\mathrm{t}=0,000 \\
p=1,000\end{array}$ \\
\hline
\end{tabular}

* Media \pm Desviación Estándar.

peso entre 65 a $85 \mathrm{~kg}$ y presentaban certificado de sanidad. Ninguna muestra pasó por un proceso de congelación previo. Se excluyeron aquellas muestras que presentaron anormalidades morfológicas a la inspección directa.

Las muestras fueron preparadas con el siguiente método: con ayuda de bisturí y pinza quirúrgica, se aisló la patela junto al tendón patelar, desinsertando ese último de la tuberosidad anterior de la tibia. Se midió el grosor, el ancho y se estandarizó el largo de todas las muestras en $45 \mathrm{~mm}$ desde su inserción patelar (Tabla 1). Con el objetivo de crear un anclaje óseo a la máquina de tracción, utilizando un taladro standard (Bosch ${ }^{\circledR}$ GSB 13 RE professional) y una broca de $8 \mathrm{~mm}$, se perforó la patela en sentido ánteroposterior.

Las muestras fueron asignadas de forma aleatoria en 2 grupos de estudio según la técnica de sutura a utilizar:
- Grupo 1 - Doble Bunnell con disposición perpendicular (14 muestras).

- Grupo 2 - Doble Bunnell con disposición paralela (14 muestras).

Todas las muestras fueron suturadas con material ultrarresistente (FiberWire \# $2^{\circledR}$, Arthrex, Naples, FL). Cada sutura Bunnell comenzó y finalizó en el borde libre del tendón. Se realizaron 3 pasadas separadas por $10 \mathrm{~mm}$ entre sí (-Fig. 1). Las muestras suturadas fueron conservadas por 24 horas a $4^{\circ}$ grados Celsius, embebidas en suero fisiológico y envueltas en tejido graso y muscular remanentes del mismo animal.

\section{Implementación}

La resistencia del material fue evaluada a través del punto máximo de fallo (PMF), utilizando una máquina computarizada de tracción, con un transductor de fuerza incorporado (Universal Stress-Strain Machine). Las muestras fueron fijadas a través de clamps especiales diseñados para evitar el deslizamiento de las suturas (-Fig. 2).

La máquina de ensayo fue calibrada previo a la medición de las muestras, utilizando pesos conocidos. Posteriormente, se aplicó tracción axial continua sobre las muestras hasta el fallo y se registró la curva de tensión. Los datos fueron registrados utilizando el Software IGOR (Versión 6.2, Wavemetrics, Lake Oswego, OR, USA). Se consideró PMF al punto más alto observado previo a la inflexión en la curva de fuerza, producida al momento del fallo de la reparación (-Fig. 3 ).

Se evaluó el modelo de falla mecánica macroscópicamente en todas las muestras.

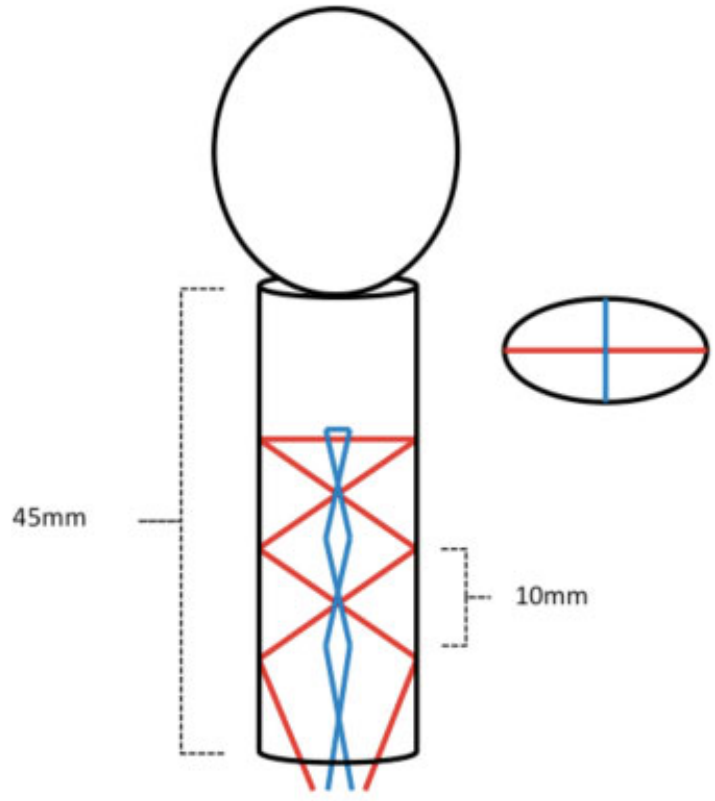

Doble Bunnell Perpendicular

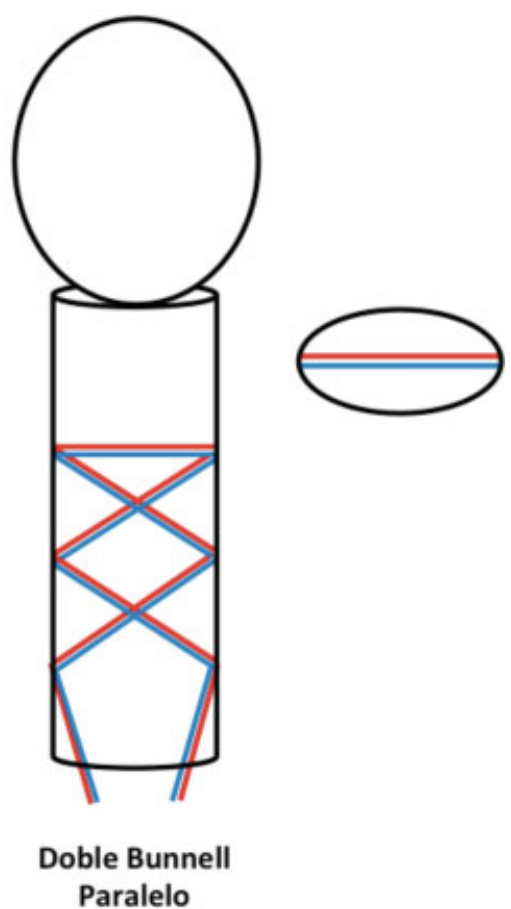

Fig. 1 Esquema de la técnica de doble Bunnell dispuesta en los 2 grupos de estudio. Cada sutura Bunnell comenzó desde el borde libre del tendón y se realizaron 3 pasadas separadas por $10 \mathrm{~mm}$ entre sí, quedando los 4 extremos de sutura en el borde libre del tendón. 


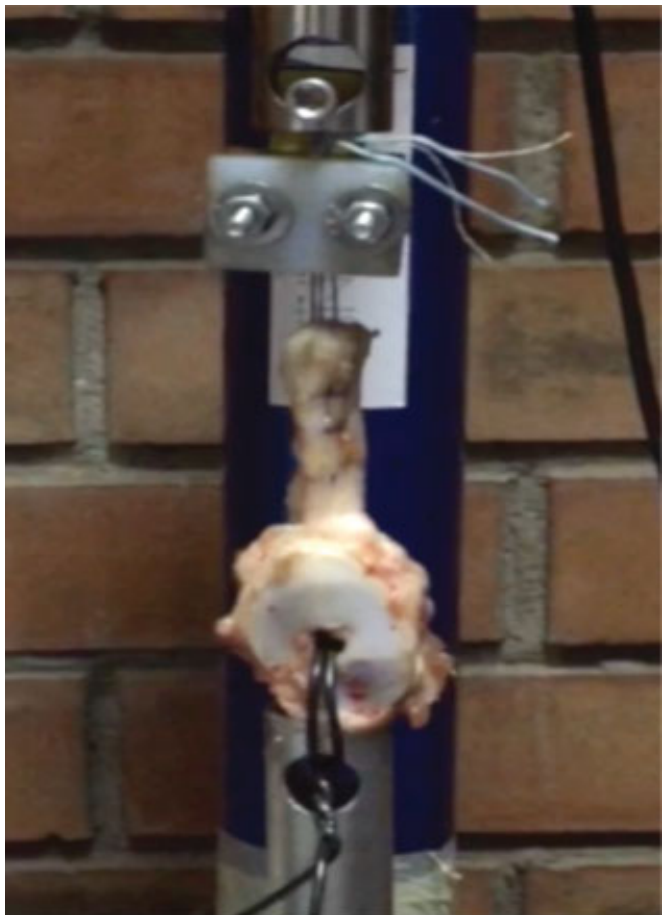

Fig. 2 Muestra fijada a la máquina de tracción. Las muestras fueron ancladas al soporte móvil de la máquina de tracción a través de un pasador trans-patelar para asegurar la firmeza del constructo. Las suturas del Bunnell fueron ancladas en un soporte fijo.

\section{Análisis Estadístico}

Para el cálculo del n muestral se utilizó una Desviación Estándar (DS) de 40 Newton (N), obtenida de un estudio biomecánico previo, ${ }^{17}$ con una diferencia de media esperada de $50 \%$, asumiendo un nivel de confianza del $95 \%$ y una potencia del $80 \%$.

Se realizó un análisis descriptivo de estadísticas de tendencia central. Para la comparación del PMF se verificó

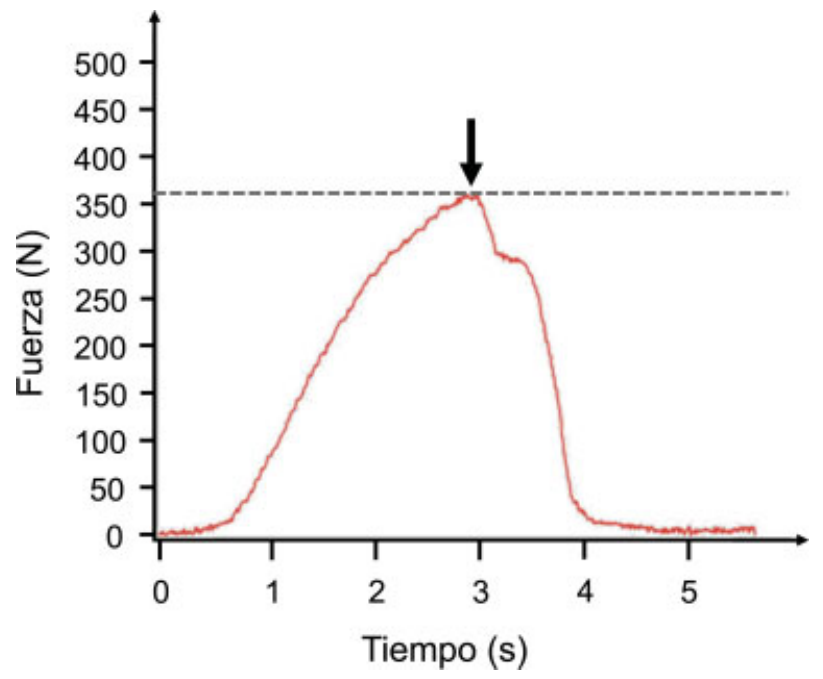

Fig. 3 Se muestra una curva de tensión representativa. El punto máximo de fallo (flecha negra), representa la resistencia máxima de la reparación previo a su rotura. distribución normal de los datos con prueba de Shapiro Wilk y luego se utilizó prueba $t$-student paramétrica de muestras independientes (no pareadas). Se consideró un valor de $p<0,05$ para determinar la significancia estadística.

Los datos se analizaron con el software estadístico SPSS versión 22.0 (SPSS Inc., Chicago, Illinois, USA) y la estimación del tamaño muestral con EPIDAT 4.1 (Software para análisis epidemiológico, OPS).

\section{Resultados}

Grupo 1: PMF promedio $=342 \pm 50,7 \quad$ N. Todas las muestras sufrieron avulsión central, preservando las fibras perimetrales.

Grupo 2: PMF promedio $=358 \pm 34,0 \quad$ N. Todas las muestras sufrieron una división longitudinal del tendón, preservando sus fibras.

No se observaron diferencias significativas entre los dos grupos $(\mathrm{t}=-0,970 ; p=0,340)$.

El modelo de falla macroscópico se aprecia en la - Fig. 4. Todas las muestras fallaron por desgarro de las fibras tendíneas. En ningún caso hubo rotura de la sutura, ni deslizamiento del anclaje, previo al fallo de la muestra.

\section{Discusión}

Lesiones que involucran una rotura del tendón patelar con insuficiencia del aparato extensor, requieren de una reparación quirúrgica con suturas. Se ha demostrado biomecánicamente, que aumentar el número de suturas dentro de cierto rango, aumenta la resistencia de una reparación tendínea, ${ }^{13,14,18}$ independiente de la técnica de sutura utilizada (Bunnell, Krackow y Kessler). Eso permite una rehabilitación acelerada que ha reportado un retorno a las actividades diarias $\mathrm{y}$ deportivas de forma precoz y segura. ${ }^{13-15,19-21}$

En un estudio biomecánico previo no publicado, demostramos con éxito esta premisa: una reparación de tendón patelar con doble Bunnell, en que las suturas fueron dispuestas perpendicularmente entre sí, fue significativamente más resistente que la reparación con un Bunnell simple. ${ }^{17}$ Es a raíz de ese estudio que surge la interrogante de si el aumento en la resistencia fue dada por la configuración perpendicular de la reparación, por el número de suturas utilizadas en cada grupo o por ambas.

En la investigación de Benjamin y col., se demostró con el punto Massive, que agregar una segunda sutura dispuesta perpendicular al punto horizontal clásico, aumenta la resistencia de la reparación para patología del manguito rotador, argumentando que se genera un mecanismo de anclaje entre ambas suturas. ${ }^{22}$ Respecto al aparato extensor de rodilla, a nuestro entender, hasta la fecha no hay estudios publicados sobre la relación entre la disposición de las suturas y la resistencia de la reparación con técnicas de doble sutura.

La hipótesis de trabajo fue que una disposición perpendicular sería más resistente al fallo que una disposición paralela por dos motivos: en primer lugar, debido a que proporcionaría un sostén adicional ante la 


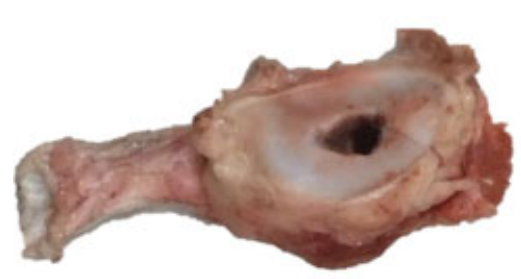

Grupo 1: Perpendicular

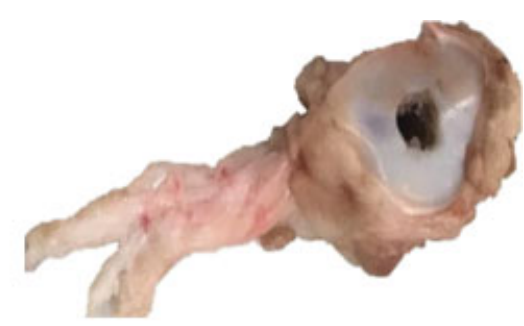

Grupo 2: Paralelo

Fig. 4 Se observa el fallo macroscópico de una muestra representativa para cada grupo de estudio. En el grupo 1, todas las muestras sufrieron avulsión central, preservando las fibras perimetrales. En el grupo 2, todas las muestras sufrieron una división longitudinal del tendón.

eventual falla de una de las 2 suturas, generando un anclaje de una sutura sobre la otra; en segundo lugar, se sabe que la resistencia aumenta en relación directa a una mayor interface entre sutura-tendón, ${ }^{13,16}$ lo que explicaría por qué 2 suturas son más resistentes que una. Si bien la interface tendón-sutura de ambos grupos, perpendicular y paralelo, serían similares, el grupo perpendicular tendría la ventaja de que cada sutura abarcaría fibras en distintas disposiciones espaciales, mientras que las suturas paralelas compartirían fibras con la misma disposición espacial, lo que implicaría una menor resistencia.

Pese a ese beneficio teórico, los resultados de este estudio, no muestran diferencias significativas en el punto máximo de fallo entre las 2 técnicas, lo cual sugiere que en este modelo es más importante el número de suturas, que su distribución espacial en el tendón, lo cual concuerda con el estudio de Dogramaci y col. $^{8}$ Eso podría deberse a la distribución de las fibras de colágeno en el tendón patelar: dado que esas fibras son longitudinales, la tracción axial de las suturas, independiente de las técnicas evaluadas, podría generar un deslizamiento entre las fibras, divulsionando los haces de colágeno sin lograr un anclaje más efectivo en el tejido. Eso concuerda macroscópicamente con el modelo de falla biomecánico en el que todas las muestras fallaron por un desgarro longitudinal de los tendones ("pull out").

Respecto de la resistencia al punto máximo de fallo de las muestras encontrado en nuestro estudio (alrededor de $350 \mathrm{~N}$ ), ella es sustancialmente mayor que el de otros estudios mecánicos similares con técnicas simples. ${ }^{16,23}$ Esa diferencia probablemente se debe a que en nuestro estudio, ambos grupos utilizaron una técnica con doble sutura. ${ }^{8}$ McCoy y col., sin embargo, utilizaron dentro de uno de sus grupos de estudio, una técnica de Bunnell doble paralelo en tendón de Aquiles humano, obteniendo un promedio inferior a $200 \mathrm{~N}$ de resistencia final, muy inferior a nuestros resultados. ${ }^{13}$ Dentro de su modelo de falla destaca que todas sus muestras fallaron a nivel de los nudos de sutura, sin dar una explicación al respecto. Sería una fortaleza de nuestro estudio que ninguno de los nudos falló y por tanto la resistencia final fue mayor.

Si bien no era un objetivo de este estudio, es interesante mencionar el hallazgo de que en todas las muestras quedó un remanente de tejido posterior al fallo. En un escenario clínico, ese remanente de tendón podría permitir una nueva reparación primaria sin necesidad de injerto, independiente de la configuración espacial del doble Bunnell. Eso sería una ventaja respecto de la falla de otros métodos de reparación como el punto de Krackow, que amputa el tejido al momento del fallo. ${ }^{17}$

Dentro de las fortalezas de este estudio podemos decir que, a nuestro entender, este es el primer estudio biomecánico que indaga la relación de la disposición espacial de la segunda sutura en aparato extensor de rodilla. Se utilizó un modelo biomecánico validado previamente en un estudio no publicado $^{17}$ en el que todas las muestras en cada grupo de estudio fallaron de igual manera.

Respecto a las debilidades de este estudio, se reconoce que el modelo biomecánico fue realizado en tendones de porcino, que si bien son similares al tendón patelar de humano, no tienen exactamente la misma morfología. No obstante, el uso de modelos con tendones animales en la literatura, ha sido ampliamente validado por su gran disponibilidad, costo y similitud al tejido humano. ${ }^{23-25}$ Otro aspecto a considerar, es que en nuestro modelo sólo se aplicaron cargas axiales continuas, que difieren de las cargas fisiológicas cíclicas y angulares, a las que se vería expuesta una reparación en una rodilla humana. Pese a esto, dado que el objetivo de nuestro estudio era comparar la resistencia a través del punto máximo de fallo, sin explorar otras propiedades mecánicas de la reparación, no fue necesario aplicar otro tipo de cargas al modelo.

Los resultados de este estudio, refutan la hipótesis de trabajo: no se encontraron diferencias significativas entre la disposición perpendicular y paralela del doble Bunnell.

\section{Conclusión}

La resistencia de la reparación de un tendón patelar con doble Bunnell perpendicular o paralelo, no mostró diferencias significativas en este modelo biomecánico.

Declaración de Conflictos de Interés

No existen conflictos que declarar para el desarrollo de este trabajo. El trabajo fue financiado a través de un fondo docente de la Universidad del Desarrollo. 


\section{Referencias}

1 Melvin JS, Karunakar MA. Patella Fractures and Extensor Mechanisim Injuries. In: Charles M, Heckman J, McQueen M, et al, editors. Rockwood and Green's Fractures in Adults. 8th Edition. Philadelphia: Wolters Kluwer Health; 2015:2269-2302

2 Zernicke RF, Garhammer J, Jobe FW. Human patellar-tendon rupture. J Bone Joint Surg Am 1977;59(02):179-183

3 Larsen E, Lund PM. Ruptures of the extensor mechanism of the knee joint. Clinical results and patellofemoral articulation. Clin Orthop Relat Res 1986;(213):150-153

4 Marder RA, Timmerman LA. Primary repair of patellar tendon rupture without augmentation. Am J Sports Med 1999;27(03):304-307

5 Ramseier LE, Werner CM, Heinzelmann M. Quadriceps and patellar tendon rupture. Injury 2006;37(06):516-519

6 Lee D, Stinner D, Mir H. Quadriceps and patellar tendon ruptures. J Knee Surg 2013;26(05):301-308

7 Gilmore JH, Clayton-Smith ZJ, Aguilar M, Pneumaticos SG, Giannoudis PV. Reconstruction techniques and clinical results of patellar tendon ruptures: Evidence today. Knee 2015;22(03):148-155

8 Dogramaci Y, Kalaci A, Sevinç TT, Esen E, Komurcu M, Yanat AN. Does strand configuration and number of purchase points affect the biomechanical behavior of a tendon repair? A biomechanical evaluation using different kessler methods of flexor tendon repair. Hand (NY) 2008;3(03):266-270

9 Aoki M, Pruitt DL, Kubota H, Manske PR. Effect of suture knots on tensile strength of repaired canine flexor tendons. J Hand Surg [Br] 1995;20(01):72-75

10 Barrie KA, Wolfe SW, Shean C, Shenbagamurthi D, Slade JF III, Panjabi MM. A biomechanical comparison of multistrand flexor tendon repairs using an in situ testing model. J Hand Surg Am 2000;25(03):499-506

11 Waitayawinyu T, Martineau PA, Luria S, Hanel DP, Trumble TE. Comparative biomechanic study of flexor tendon repair using FiberWire. J Hand Surg Am 2008;33(05):701-708

12 Winters SC, Gelberman RH, Woo SL, Chan SS, Grewal R, Seiler JG III. The effects of multiple-strand suture methods on the strength and excursion of repaired intrasynovial flexor tendons: a biomechanical study in dogs. J Hand Surg Am 1998;23(01):97-104

13 McCoy BW, Haddad SL. The strength of achilles tendon repair: a comparison of three suture techniques in human cadaver tendons. Foot Ankle Int 2010;31(08):701-705
14 Rawson S, Cartmell S, Wong J. Suture techniques for tendon repair; a comparative review. Muscles Ligaments Tendons J 2013;3(03):220-228

15 Watson TW, Jurist KA, Yang KH, Shen KL. The strength of Achilles tendon repair: an in vitro study of the biomechanical behavior in human cadaver tendons. Foot Ankle Int 1995;16 (04):191-195

16 Gebauer M, Beil FT, Beckmann J, et al. Mechanical evaluation of different techniques for Achilles tendon repair. Arch Orthop Trauma Surg 2007;127(09):795-799

17 Vaisman A, Melean P, Figueroa D, Figueroa F, Calvo R. Villalón. Biomechanical evaluation of the Bunnell vs. Modified Bunnell suture: An experimental study in porcine ex vivo patellar tendons. Orthopeadic Proceedings. Bone Joint Res. 2011; (SUPPII): 116. Disponible en: http://www.bjjprocs.boneandjoint. org.uk/content/93-B/SUPP_II/116.3

18 Gelberman RH, Manske PR. Factors influencing flexor tendon adhesions. Hand Clin 1985;1(01):35-42

19 Strickland JW. Development of flexor tendon surgery: twentyfive years of progress. J Hand Surg Am 2000;25(02):214-235

20 Mandelbaum BR, Myerson MS, Forster R. Achilles tendon ruptures. A new method of repair, early range of motion, and functional rehabilitation. Am J Sports Med 1995;23(04):392-395

21 West JL, Keene JS, Kaplan LD. Early motion after quadriceps and patellar tendon repairs: outcomes with single-suture augmentation. Am J Sports Med 2008;36(02):316-323

22 Ma CB, MacGillivray JD, Clabeaux J, Lee S, Otis JC. Biomechanical evaluation of arthroscopic rotator cuff stitches. J Bone Joint Surg Am 2004;86-A(06):1211-1216

23 Jordan MC, Hoelscher-Doht S, Fehske K, Gilbert F, Jansen H, Meffert RH. Bunnell or cross-lock Bunnell suture for tendon repair? Defining the biomechanical role of suture pretension. J Orthop Surg 2015;10:192-200

24 Gil F, Ramírez G, Ayala M, et al. Anatomía interactiva del cerdo. Universidad de Murcia. España. (Leído 20 Mar 2014). Disponible en: https://www.um.es/anatvet/interactividad/acerdo/Anatom\% EDa\%20Interactiva\%20del\%20Cerdo.pdf

25 Orrego M, Matas J, Abusleme S, Guzmán-Venegas R, Amenabar D. Effect of cyclic tension on the biomechanical properties of flexor tendon grafts. Results of an ex-vivo porcine study. Knee 2014;21 (06):1029-1032 